\title{
Reversal of multidrug resistance in
} human lung cancer cells by delivery of 3-octadecylcarbamoylacrylic acid-cisplatin-based liposomes

\author{
This article was published in the following Dove Press journal: \\ Drug Design, Development and Therapy \\ 17 February 2017 \\ Number of times this article has been viewed
}

\author{
Juan Song \\ Weifang Ren \\ Tingting $\mathrm{Xu}$ \\ Yi Zhang \\ Hongyu Guo \\ Shanshan Zhu \\ Li Yang
}

Department of Pharmaceutics, College of Pharmacy, Shenyang Pharmaceutical University, Shenyang, Liaoning, People's Republic of China
Correspondence: Li Yang Department of Pharmaceutics, College of Pharmacy, Shenyang Pharmaceutical University, 103 Wenhua Road, Shenyang, Liaoning I I00 I6, People's Republic of China $\mathrm{Tel} / \mathrm{fax}+862423986349$ Email pharm305@I26.com

\begin{abstract}
Liposome-based drug delivery system would be an innovative and promising candidate to circumvent multidrug resistance (MDR) of cisplatin (CDDP). However, the reversal efficacy of liposomal CDDP was severely impaired by weak cellular uptake and insufficient intracellular drug release. In this study, 3-octadecylcarbamoylacrylic acid-CDDP nanocomplex (OMI-CDDP-N)-based liposomes (OCP-L) with high cellular uptake and sufficient intracellular drug release were designed to circumvent MDR of lung cancer. OMI-CDDP-N was synthesized through a $\mathrm{pH}$-sensitive monocarboxylato and an $\mathrm{O} \rightarrow \mathrm{Pt}$ coordinate bond, which is more efficient than CDDP. Also, OCP-L incorporated with OMI-CDDP-N could induce effective cellular uptake, enhanced nuclear distribution, and optimal cellular uptake kinetics. In particular, OCP-L presented superior effects on enhancing cell apoptosis and in vitro cytotoxicity in CDDP-resistant human lung cancer (A549/CDDP) cells. The mechanisms of MDR reversal in A549/CDDP cells by OCP-L could attribute to organic cation transporter 2 restoration, ATPase copper-transporting beta polypeptide suppression, hypoxia-inducible factor $1 \alpha$-subunit depletion, and phosphatidylinositol 3-kinase/Akt pathway inhibition. These results demonstrated that OCP-L may provide an effective delivery of CDDP to resistant cells to circumvent MDR and enhance the therapeutic index of the chemotherapy.
\end{abstract}

Keywords: 3-octadecylcarbamoylacrylic acid-cisplatin nanocomplexes, liposomes, cellular uptake, multidrug resistance, therapeutic index

\section{Introduction}

Lung cancer is the most common malignancy and the leading cause of death worldwide. ${ }^{1}$ It is also the most frequently diagnosed cancer in men, accounting for $16.7 \%$ of the total cancer cases. ${ }^{2}$ For the efficient treatment of lung cancer, cisplatin (CDDP)-based chemotherapy has been demonstrated as the backbone in clinics. ${ }^{3}$ Unfortunately, the success of chemotherapy is hindered by severe systemic toxicity and the development of multidrug resistance (MDR). MDR of CDDP can result from numerous mechanisms, which may be divided into the following categories: decreased cellular uptake of CDDP, increased cellular efflux of CDDP, increased DNA repair, and altered drug inactivation. ${ }^{4}$

Liposome can effectively bypass drug efflux through nonspecific or specific endocytosis, which suggests the potential for MDR reversal. ${ }^{5,6}$ Interestingly, only a few studies have reported about the use of liposomal CDDP to circumvent tumor resistance, and the corresponding mechanisms still remain elusive. ${ }^{7,8}$ More importantly, 
the weak cellular uptake and insufficient intracellular drug release of liposomal CDDP present a major obstacle for MDR reversal. For instance, Carvalho Júnior et $\mathrm{al}^{9}$ showed that stealth $\mathrm{pH}$-sensitive liposomes were effective in GLC4/ CDDP. However, dense hydrophilic poly(ethylene glycol) shells hindered the penetration and endocytosis of these stealth $\mathrm{pH}$-sensitive liposomes, resulting in weak cellular uptake. Moreover, the resistant mechanisms of these stealth pH-sensitive liposomes are still unknown. SPI-077, a sterically stabilized liposomal CDDP, exhibited MDR phenomena and poor clinical efficacy because of the insufficient intracellular drug release. ${ }^{10,11}$

To resolve the aforementioned problems, 3-octadecylcarbamoylacrylic acid-CDDP nanocomplex (OMI-CDDPN)-based liposomes (OCP-L) were designed to circumvent the MDR of CDDP. The criticality factor of OCP-L in effectively circumventing MDR was the introduction of a functional OMI. First, OMI had the intrinsic ability to chelate active platinum through an acid-labile monocarboxylato and an $\mathrm{O} \rightarrow \mathrm{Pt}$ coordinate bond. Previously, we demonstrated that OMI-CDDP-N was more efficient than CDDP because of the unstable monocarboxylato and an $\mathrm{O} \rightarrow \mathrm{Pt}$ coordinate bond. ${ }^{12}$ Second, the "perturbation" effect of OMI could increase the permeability on the cell membrane, resulting in much more intercellular uptake of active platinum and OCP-L. Third, the lipophilic octadecyl tail of OMI had a notable inhibition effect on the expression of efflux transporters, which could increase accumulation and suppress active platinum efflux in drug-resistant cancer cells. Furthermore, once uptaken into the acidic environment of the endosome, the OCP-L could efficiently release active platinum into cytoplasm to exert therapeutic effect because of the acid-labile monocarboxylato and an $\mathrm{O} \rightarrow \mathrm{Pt}$ coordinate bond. In this study, the potential of OCP-L in reversing MDR was evaluated in a CDDPresistant human lung cancer cell line (A549/CDDP). The cellular uptake and distribution, cellular uptake kinetics, cell apoptosis, and in vitro cytotoxicity were observed in A549/ CDDP and compared with commercial CDDP injection (CDDP-S). In addition, the possible mechanisms of OCP-L to reverse MDR were also performed.

\section{Materials and methods \\ Materials}

OMI-CDDP-N was previously synthesized and characterized by our group. ${ }^{12}$ CDDP-S was obtained from Qilu Pharmaceutical Co., Ltd. (Shandong, People's Republic of China). 1,1-Dioctadecyl-3,3,3,3-tetramethylindotricarbocyanine iodide (Dir) (excitation wavelength: $748 \mathrm{~nm}$; emission wavelength: $780 \mathrm{~nm}$ ) was obtained from Fanbo Biochemical
Co., Ltd. (Beijing, People's Republic of China). Chlorpromazine and nystatin were obtained from Dalian Meilun Biotech Co., Ltd. (Dalian, People's Republic of China). Annexin V-APC/7-AAD apoptosis assay kit was purchased from KeyGEN Biotech Co., Ltd. (Nanjing, People's Republic of China). All other reagents were of analytical grade and used without further purification.

\section{Tumor cell lines and cell culture}

A549 and A549/CDDP were purchased by Nanjing KeyGEN Biotech Co., Ltd. (Nanjing, People's Republic of China). A549 and A549/CDDP cells were cultured in Roswell Park Memorial Institute 1640 media (Thermo Fisher Scientific, Waltham, MA, USA), supplemented with 10\% fetal bovine serum (Shanghai ExCell Biology Inc., People's Republic of China) and penicillin-streptomycin solution at $37^{\circ} \mathrm{C}$ with $5 \% \mathrm{CO}_{2}$. A549/CDDP cells were cross-resistant to some other drugs. ${ }^{13}$

\section{Preparation of Dir-labeled OCP-L}

OCP-L was prepared and characterized according to the method previously reported by our group. ${ }^{12}$ The Dir-labeled OCP-L was also prepared as OCP-L. Briefly, Dir, soybean phospholipids, and cholesterol were dissolved in organic solvent and then an aqueous solution containing OMICDDP-N was added to the lipid mixture. This mixture was then immediately emulsified with a probe-type sonicator (JY92-2D Sonicator, Xinzhi Biotech Co., Ltd., Ningbo, People's Republic of China) to form water/oil-type emulsion. The emulsion was dried down to a semi-solid gel in a rotary evaporator at $40^{\circ} \mathrm{C}$. The volume of the resulting gel was then adjusted with aqueous solution, and the resulting liposome was sterilized by passing through a $0.22-\mu \mathrm{m}$ filter. The blank liposomes (OP-L) were also prepared as described for Dirlabeled OCP-L except for the absence of Dir and CDDP.

\section{Cell uptake}

To measure the amount of platinum in cells, A549/CDDP cells $\left(1 \times 10^{5}\right.$ cells/well) were treated with OCP-L-1 (OMI: 0.4\%), OCP-L-2 (OMI: 0.6\%), OCP-L-3 (OMI: 0.8\%), or CDDP-S at a CDDP concentration of $4 \mu \mathrm{M}$ for $48 \mathrm{~h}$. After that, the cells were rinsed and lysed. Then, the cell lysates were digested in $\mathrm{H}_{2} \mathrm{O}_{2}\left(30 \%\right.$ vol \%) and $\mathrm{HNO}_{3}(68 \%$ vol \%) and diluted in water to a final acid content of $5 \%$. The bicinchoninic acid protein assay kit was used for measuring the protein amount, while the platinum concentration was determined by 7900 inductively coupled plasma mass spectrometer (ICP-MS) from Agilent Technologies (CA, USA). The data of protein amount and platinum concentration were 
determined three times. The cellular uptake was expressed as ng of platinum/mg of protein.

For high-content analysis, A549/CDDP cells were treated with Dir-labeled OCP-L-1 and OCP-L-3 at a CDDP concentration of $4 \mu \mathrm{M}$ for $48 \mathrm{~h}$. The intracellular distribution of Dir-labeled OCP-L was observed in the high-content analysis images (Image Xpress Micro, USA) obtained after staining nucleus with 4',6-diamidino-2-phenylindole (DAPI).

\section{Cellular uptake kinetics of OCP-L}

The A549/CDDP cells were seeded in six-well plates for $24 \mathrm{~h}$ and incubated with OCP-L-3 or CDDP-S for $15 \mathrm{~min}$ and 2, 4, 8, 12, 24, and 48 h, respectively. The treated A549/ CDDP cells were rinsed three times with phosphate-buffered saline (PBS) and then harvested by trypsinization. After the cells were collected, the platinum concentration in the cells was determined by ICP-MS three times.

\section{Endocytosis pathway}

The A549/CDDP cells $\left(1 \times 10^{5}\right.$ cells/well $)$ were seeded in six-well plates for $24 \mathrm{~h}$. After incubation with the specific endocytosis inhibitors for $2 \mathrm{~h}$ at $37^{\circ} \mathrm{C}$, the Dir-labeled OCP-L-3 was added to each well of the culture dish. The blank control cells were incubated with the same concentration of Dir-labeled OCP-L without the endocytosis inhibitors. The obtained single-cell suspension was identified by a Becton Dickinson and Company, FACS Calibur Cytometer (Franklin Lakes, NJ, USA).

\section{Cell apoptosis determination}

A549/CDDP cells were seeded in six-well plates and treated with CDDP-S or OCP-L-3 at a CDDP concentration of $32 \mu \mathrm{M}$ for $48 \mathrm{~h}$. After incubation, the cells were washed twice with PBS and suspended in 500- $\mu \mathrm{L}$ binding buffer. The untreated cells were used as the control. For measurement of apoptosis, cells were stained with $5-\mu \mathrm{L}$ Annexin V-APC and $5-\mu \mathrm{L} 7-\mathrm{AAD}$ according to the manufacturer's instruments. Cell apoptosis data were analyzed using a Becton Dickinson FACS Calibur Cytometer.

\section{MDR reversal by cytotoxicity assay}

The MTT assay was performed to evaluate the chemosensitivity of OCP-L in A549 and A549/CDDP cell lines. The cells were plated in 96 -well plates at a density of $5 \times 10^{3}$ cells/well. After $24 \mathrm{~h}$, the cells were treated with varying concentrations of CDDP-S or OCP-L-3 and incubated for $48 \mathrm{~h}$. The culture medium was replaced by MTT solution $(5 \mathrm{mg} / \mathrm{mL}$, $20 \mu \mathrm{L} /$ well). After incubation for $4 \mathrm{~h}$, the medium was removed and $150 \mu \mathrm{L}$ of dimethyl sulfoxide solution was added. The absorbance values were measured at $490 \mathrm{~nm}$ using a microplate reader (Spectra Max M3, Molecular Devices, USA). This experiment was repeated three times.

\section{Western blotting analysis}

A549/CDDP cells were collected after $48 \mathrm{~h}$ of treatment with $32 \mu \mathrm{M}$ CDDP-S or OCP-L-3 and processed for protein extraction and Western blotting using standard procedures as described earlier. ${ }^{14}$ The protein lysates were separated in precast polyacrylamide gels containing sodium dodecyl sulfate and then transferred onto nitrocellulose membranes. The membranes were blocked with a blocking buffer containing 5\% (w/v) skimmed milk. After washing thrice with Tris-buffered saline with Tween 20, the membranes were further incubated with a primary specific antibody at $4^{\circ} \mathrm{C}$. After washing, the membranes were incubated with secondary antibody (KeyGEN Biotech, Nanjing, People's Republic of China). Protein bands were visualized using the G: BOX chemiXR5 and analyzed with Gel-Pro32 (Nanjing KeyGEN Biotech).

\section{Results \\ Cell uptake}

To confirm that the OMI could facilitate cell uptake of OCP-L, the cellular uptake of CDDP in A549/CDDP cells was measured by ICP-MS. Compared with CDDP-S, a significantly improved cellular uptake was found in the A549/CDDP cells after treatment with OCP-L. As shown in Figure 1A and Table 1, OCP-L-3 delivering CDDP showed a 5.1-fold increase in drug internalization over CDDP-S (32.02 $\pm 0.53 \mathrm{ng} / \mathrm{mg}$ protein vs $164.24 \pm 1.96 \mathrm{ng} / \mathrm{mg}$ protein, $P<0.001)$. It is worth mentioning that the cellular uptake of OCP-L was related to the concentration of OMI, and the highest uptake efficiency was observed in OCP-L-3 with an OMI concentration of $0.8 \%$.

The cellular distribution of OCP-L was examined by high-content analysis, and the images are shown in Figure 1B. The green part indicated the Dir-labeled OCP-L, and the blue part represented the nucleus stained by DAPI. When the concentration of OMI was $0.4 \%$, most of the green fluorescence was observed inside the cytoplasm and only a spot of green fluorescence was seen inside the nucleus. Interestingly, a stronger green fluorescent intensity was observed inside the nucleus after incubation with OCP-L-3, which indicated that this internalized OCP-L-3 could penetrate into the nucleus.

\section{Cellular uptake kinetics of OCP-L}

There are several striking differences in cellular uptake kinetics properties between the CDDP-S and OCP-L-3. As shown in Figure 2A, the maximum amount of intracellular 
A

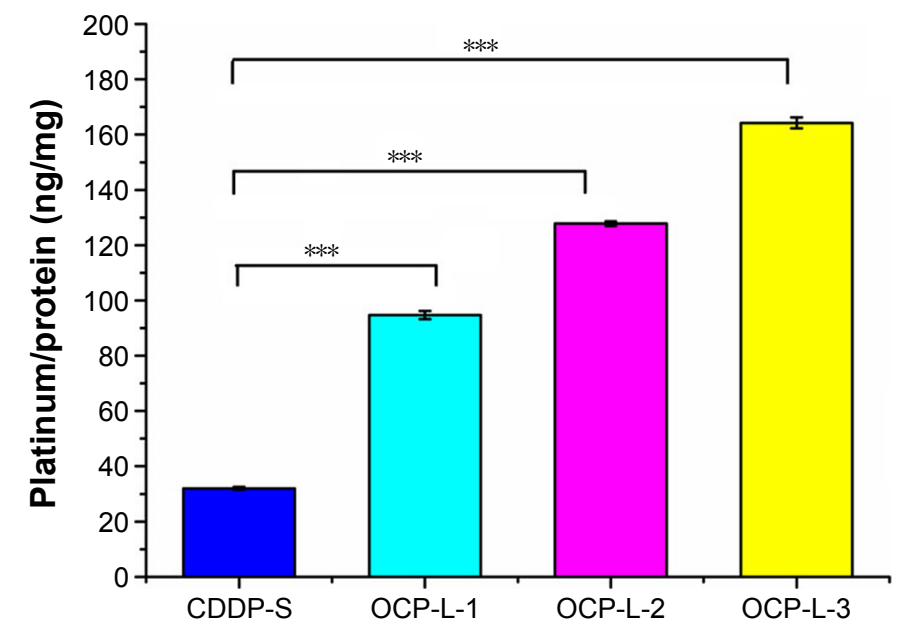

Formulations

B

Dir-labeled
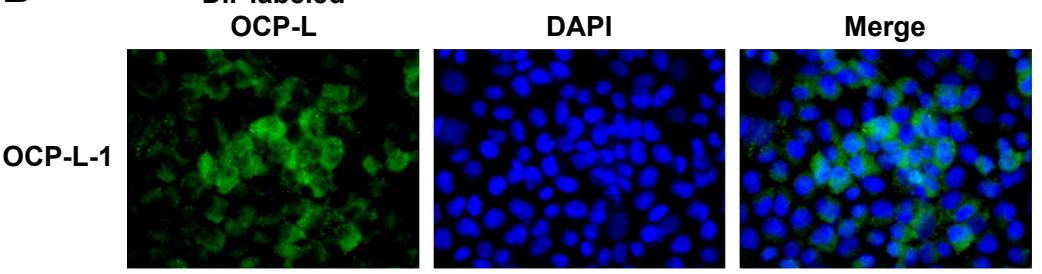

OCP-L-3
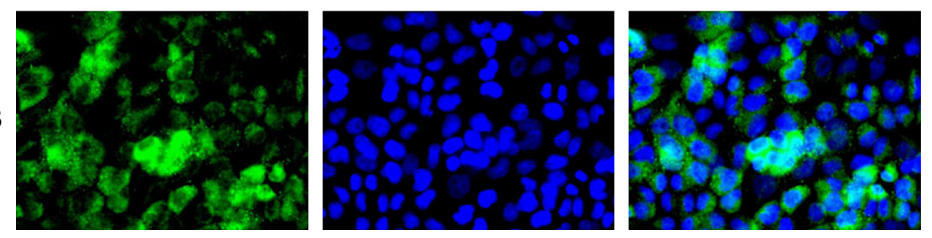

Figure I (A) In vitro cellular uptake of OCP-L with different OMI concentrations against A549/CDDP cells after $48 \mathrm{~h}$. (B)-High-content analysis images of A549/CDDP cells after incubating with Dir-labeled OCP-L (magnification 400 -fold). Data are presented as the mean \pm standard deviation. $* * * P<0.001$. The OCP-L was labeled with Dir (green), and nucleus (blue) was stained by DAPI.

Abbreviations: A549/CDDP, CDDP-resistant human lung cancer cells; CDDP, cisplatin; CDDP-S, commercial CDDP injection; Dir, I,I-dioctadecyl-3,3,3, 3-tetramethylindotricarbocyanine iodide; OCP-L, 3-octadecylcarbamoylacrylic acid-CDDP nanocomplexes-based liposomes; DAPI, 4',6-diamidino-2-phenylindole; OMI, 3-octadecylcarbamoylacrylic acid.

platinum in the CDDP-S group was $78.93 \pm 6.50 \mathrm{ng} / \mathrm{mg}$ protein, while in the OCP-L group it was about $210.80 \pm 1.17 \mathrm{ng} / \mathrm{mg}$ protein $(P<0.001)$. After $2 \mathrm{~h}$, the amount of intracellular platinum in the CDDP-S group decreased gradually. On the contrary, treatment of cells with OCP-L resulted in a gradual

Table I In vitro cellular uptake of OCP-L with different OMI concentrations against A549/CDDP cells after $48 \mathrm{~h}$

\begin{tabular}{llll}
\hline Formulations & Lipid composition & $\begin{array}{l}\text { Mass } \\
\text { ratio }\end{array}$ & $\begin{array}{l}\text { Pt content } \\
\text { (ng/mg protein) }\end{array}$ \\
\hline CDDP-S & - & - & $32.02 \pm 0.53$ \\
OCP-L-I & OMI:SPC:cholesterol & $0.6: 10: 0.7$ & $94.74 \pm 1.49 * * *$ \\
OCP-L-2 & OMI:SPC:cholesterol & $0.9: 10: 0.7$ & $127.82 \pm 0.85^{* * *}$ \\
OCP-L-3 & OMI:SPC:cholesterol & $1.2: 10: 0.7$ & $164.24 \pm 1.96 * * *$ \\
\hline
\end{tabular}

Note: $* * * P<0.001$ compared with CDDP-S.

Abbreviations: A549/CDDP, CDDP-resistant human lung cancer cells; CDDP, cisplatin; CDDP-S, commercial CDDP injection; OCP-L, 3-octadecylcarbamoylacrylic acid-CDDP nanocomplexes-based liposomes; OMI, 3-octadecylcarbamoylacrylic acid; SPC, soybean phospholipids. decrease in the amount of intracellular platinum from 2 to $8 \mathrm{~h}$, and then reached a steady state from 12 to $48 \mathrm{~h}$. According to these results, OCP-L was taken up by A549/CDDP cells more efficiently than CDDP-S. The cellular uptake of OCP-L was 2.7- to 5.4-fold higher than those of CDDP-S at 2 and $48 \mathrm{~h}$. Moreover, the area under the curve value of OCP-L was 2.8 -fold higher than that of CDDP-S $(2,399.73 \mathrm{ng} \cdot \mathrm{h} / \mathrm{mg}$ protein vs $6,751.13 \mathrm{ng} \cdot \mathrm{h} / \mathrm{mg}$ protein).

\section{Endocytosis pathways}

To identify the uptake mechanisms involved in the cellular uptake of OCP-L, several specific inhibitors were employed to block the corresponding endocytic pathway (Figure 2B). Chlorpromazine dissociates clathrin from the surface membrane to inhibit the clathrin-mediated endocytosis. When the cells were preincubated with chlorpromazine, the uptake of OCP-L was reduced by $5.99 \%$. Similarly, when the cells 
A

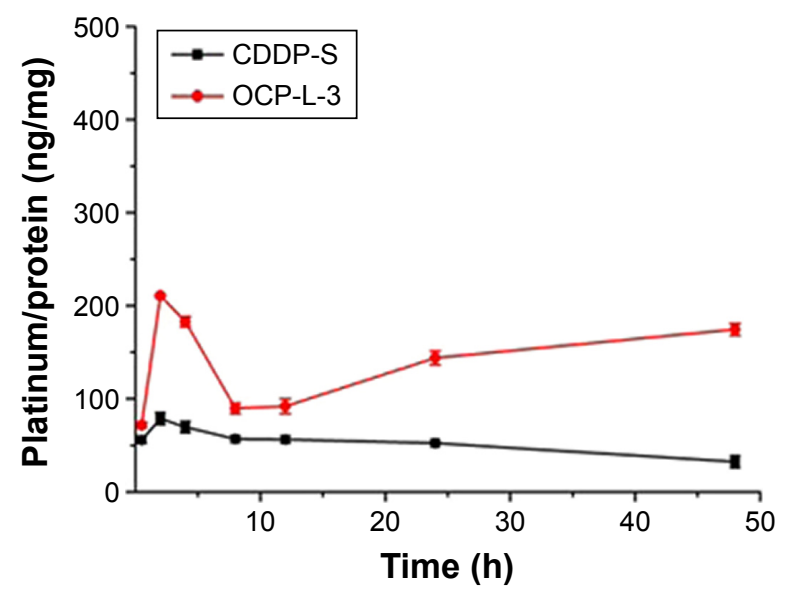

B
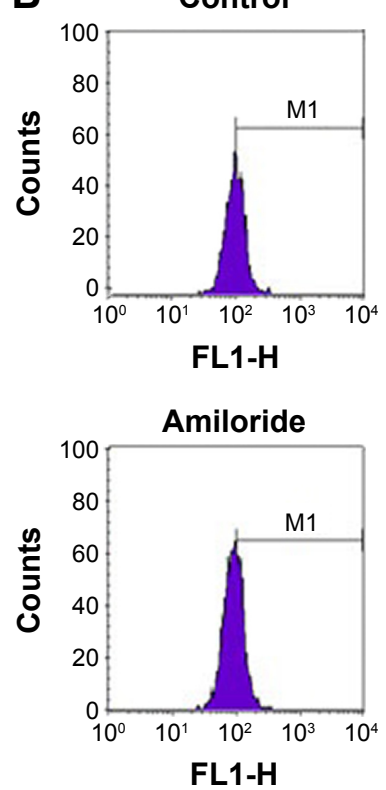

Chlorpromazine
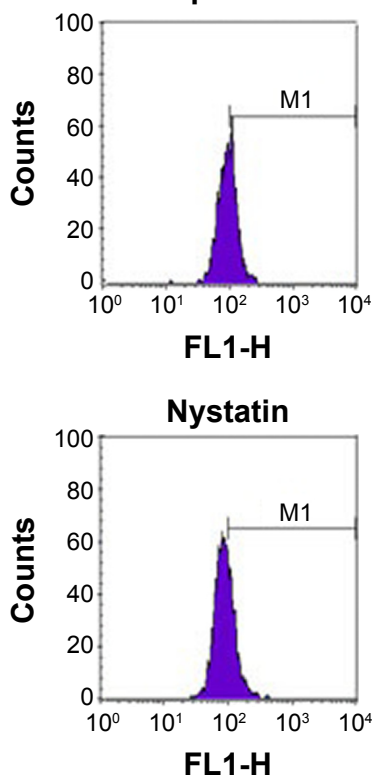

C

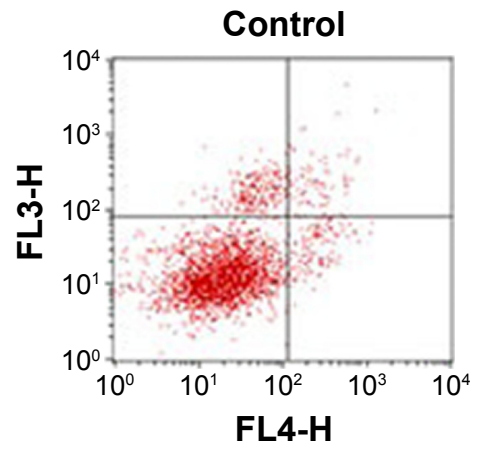

CDDP-S

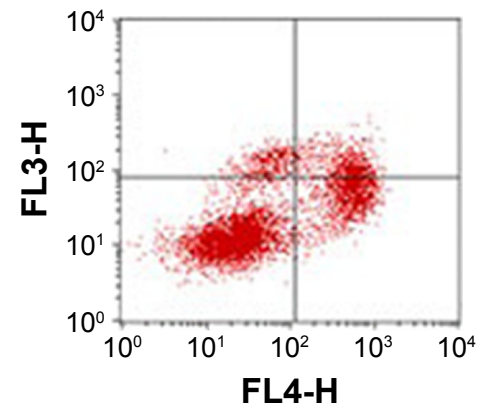

OCP-L-3

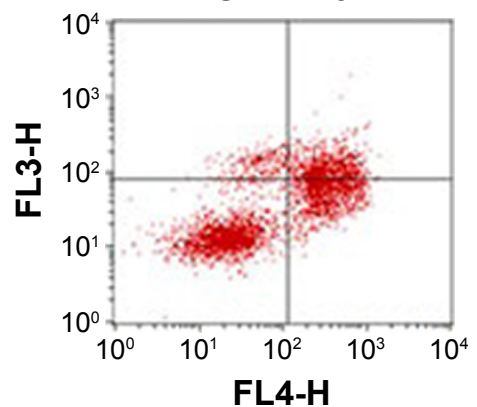

Figure 2 (A) Total cellular platinum concentration in A549/CDDP cells after incubation with OCP-L-3 or CDDP-S at different time points. Data are presented as the mean \pm standard deviation. (B) Relative cellular uptake of Dir-labeled OCP-L in A549/CDDP cells after $2 \mathrm{~h}$ of incubation at CDDP concentration of $32 \mu \mathrm{M}$ in the presence of the specific endocytosis inhibitors was compared with that in the absence of specific endocytosis inhibitors (control). (C) Apoptosis of A549/CDDP cells induced by $32 \mu M$ CDDP-S or OCP-L after $48 \mathrm{~h}$ of incubation was determined using the Annexin V-APC/7-AAD staining. Each quadrant represents the percentage of cells in early apoptosis (lower right), late apoptosis (upper right), necrosis (upper left), and healthy cells (lower left), respectively.

Abbreviations: A549/CDDP, CDDP-resistant human lung cancer cells; CDDP, cisplatin; CDDP-S, commercial CDDP injection; Dir, I,I-dioctadecyl-3,3,3,3tetramethylindotricarbocyanine iodide; h, hours; OCP-L, 3-octadecylcarbamoylacrylic acid-CDDP nanocomplexes-based liposomes.

were preincubated with amiloride, an inhibitor for macropinocytosis pathways, the cellular uptake of OCP-L was reduced by $10.48 \%$. Besides this, nystatin, an inhibitor for caveolin-mediated endocytosis pathway, also decreased the cellular uptake of OCP-L (reduced by $13.44 \%$ ). ${ }^{15}$

\section{Cell apoptosis determination}

As shown in Figure 2C, OCP-L had stronger apoptosisinducing capacity compared to CDDP-S. The A549/CDDP cells treated with OCP-L for $48 \mathrm{~h}$ had the total apoptotic ratio of $49.58 \%$, much higher than $30.58 \%$ of CDDP-S. Moreover, A549/CDDP cells treated with OCP-L exhibited an increase in early and/or late apoptosis compared to the CDDP-S. These results suggested that OCP-L could enhance the cytotoxicity effects in A549/CDDP cells compared with CDDP-S.

\section{MDR reversal}

The OCP-L improved the cytotoxicity of CDDP compared with CDDP-S in A549 cells and A549/CDDP. The $\mathrm{IC}_{50}$ values for CDDP-S were 6.05 and 63.57 $\mu \mathrm{M}$ in A549 and A549/ CDDP cells, respectively. Thus, a 10.5-fold higher dose of CDDP-S is required to produce an equivalent toxicity in A549/CDDP cells compared to A549 cells. However, the $\mathrm{IC}_{50}$ values for OCP-L were 4.15 and $35.80 \mu \mathrm{M}$ in $\mathrm{A} 549$ and A549/CDDP cells, respectively. OMI-CDDP-N loaded into liposome had an even lower $\mathrm{IC}_{50}$ value than CDDP-S in the resistant cells, which meant that OMI-CDDP-N-loaded 
liposomes could reverse the CDDP-resistant activity in A549/CDDP cells.

\section{Western blotting analysis}

Some researchers had stated that the resistance mechanisms of A549/CDDP were related to organic cation transporter 2 (OCT2), ATPase copper-transporting beta polypeptide (ATP7B), hypoxia-inducible factor $1 \alpha$-subunit (HIF-1 $\alpha$ ), and phosphatidylinositol 3-kinase (PI3K)/Akt pathways. Therefore, Western blot was used to confirm whether the

A

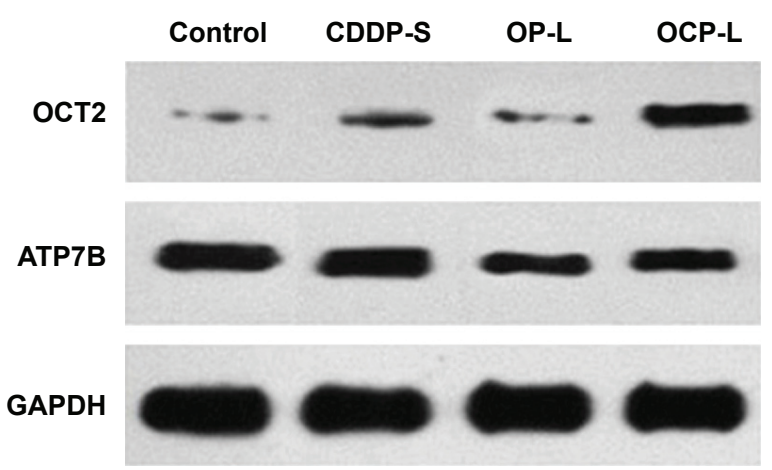

C

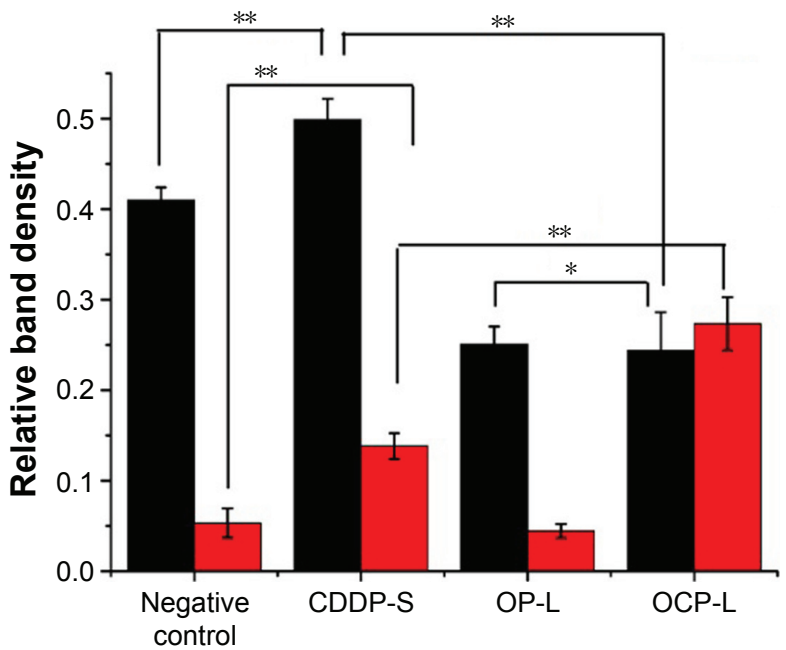

Formulations

ATP7B mechanisms of MDR reversal in A549/CDDP cells by OCP-L could attribute to OCT2, ATP7B, HIF-1 $\alpha$, and PI3K/Akt pathways. ${ }^{16-19}$ Figure $3 \mathrm{~A}, \mathrm{C}$ shows the expression level of OCT2 and ATP7B in A549/CDDP cells and the integrity ratio with glyceraldehyde-3-phosphate dehydrogenase after incubation with CDDP-S, OP-L, and OCP-L, respectively. Compared with the negative control, the expression level of OCT2 was not changed with the treatment of OP-L, but increased about 2.6-fold in the presence of CDDP-S and greatly increased about 5.2-fold when cells were treated
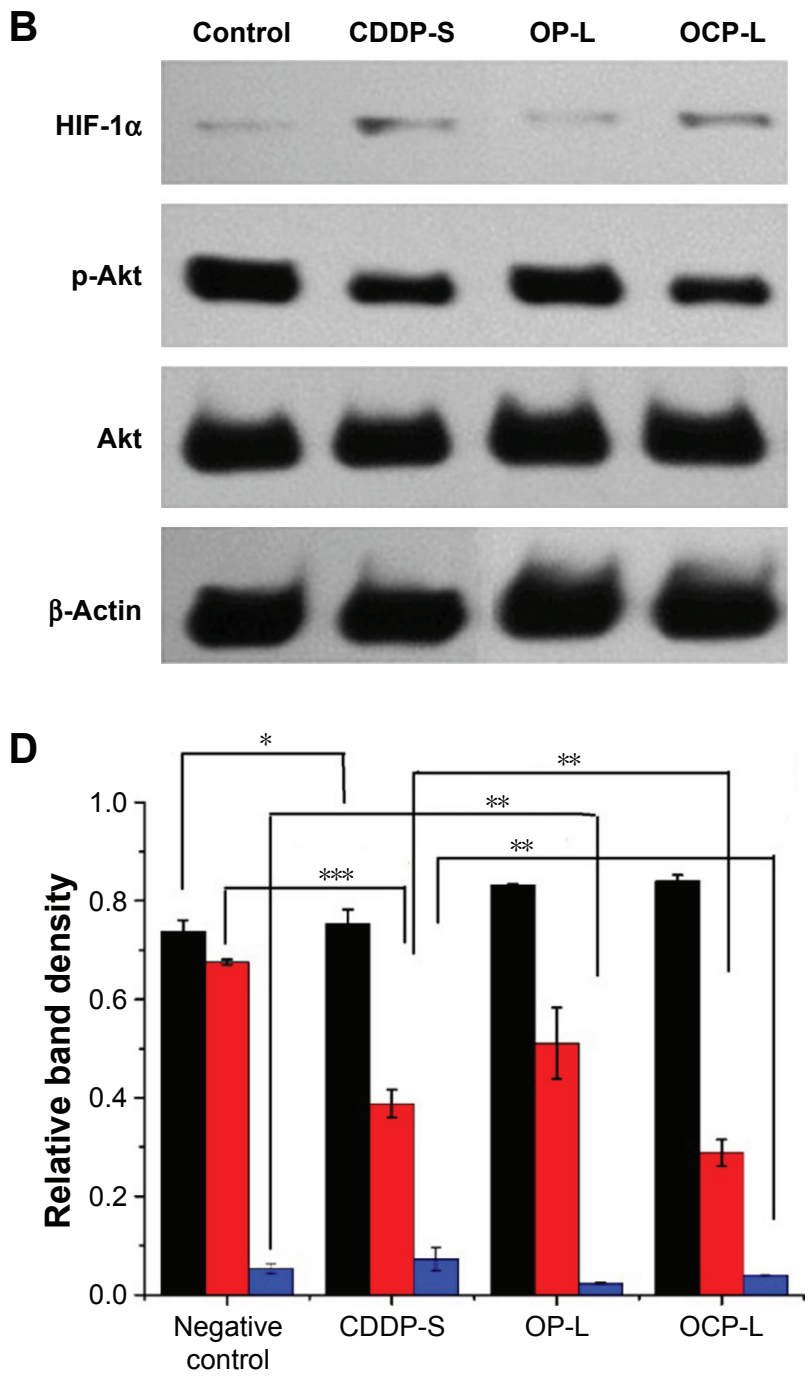

Formulations

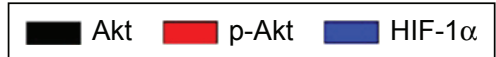

Figure 3 (A) Determination of OCT2 and ATP7B expression in A549/CDDP cells after treatment with CDDP-S, OP-L, and OCP-L for 48 h. (B) HIF-I $\alpha$, Akt, and p-Akt expressions on the A549/CDDP cells after treatment with CDDP-S, OP-L, and OCP-L for $48 \mathrm{~h}$. (C) Relative protein level of OCT2 and ATP7B. (D) Relative protein level of HIF-I $\alpha$, Akt, and p-Akt. Expressions of OCT2, ATP7B, HIF-l $\alpha$, Akt, and p-Akt were determined by Western blot. GAPDH and $\beta$-actin were used for equal loading. Data are presented as the mean \pm standard deviation $(n=2) * P>0.05, * * P<0.05$, *** $P<0.01$.

Abbreviations: A549/CDDP, CDDP-resistant human lung cancer cells; Akt, protein kinase B; ATP7B, ATPase copper-transporting beta polypeptide; HIF-I $\alpha$, hypoxiainducible factor I $\alpha$-subunit; OCT2, organic cation transporter 2; CDDP, cisplatin; CDDP-S, commercial CDDP injection; GAPDH, glyceraldehyde-3-phosphate dehydrogenase; OCP-L, 3-octadecylcarbamoylacrylic acid-CDDP nanocomplexes-based liposomes; OP-L, blank liposome. 
with OCP-L. Interestingly, compared with the negative control, the ATP7B level increased in the presence of CDDP-S but decreased when cells were treated with OCP-L, which indicated that the ATP7B expression on the surface of A549/ CDDP cells was greatly inhibited by OCP-L. Meanwhile, compared to OCP-L, the ATP7B expression in A549/CDDP cells was not obviously changed in the presence of OP-L. Therefore, the notable inhibition of ATP7B expression in A549/CDDP cells by OCP-L could attribute to the incorporation of $\mathrm{OMI}$ into the liposome.

To investigate whether OCP-L could affect the expression of HIF-1 $\alpha$ in A549/CDDP cells, the level of HIF-1 $\alpha$ was determined by Western blot. As shown in Figure 3B, D, the level of HIF-1 $\alpha$ for CDDP-S was 1.4-fold higher than that of the negative control but reduced after the treatment of OCP-L. Moreover, the HIF-1 $\alpha$ expression in A549/ CDDP cells for OCP-L was 1.7-fold higher than that of OP-L. OCP-L also exhibited inhibition effect on the p-Akt level (Figure 3B, D). Compared with negative control, the levels of p-Akt were decreased after treatment with three formulations at $48 \mathrm{~h}$. Also, cells treated with OCP-L showed the lowest level of p-Akt $(P<0.001)$, indicating that OCP-L could increase apoptosis through inhibiting the PI3K/Akt pathways in A549/CDDP cells.

\section{Discussion}

In this study, we found that OCP-L, as a novel drug delivery system, could effectively circumvent MDR phenotype in A549/CDDP cells. Figure 4 shows the schematic of OCP-L to circumvent MDR in A549/CDDP cells. OCP-L exhibited increased intracellular uptake of platinum by simultaneously exerting the "perturbation" effect of OMI and endocytosis pathway of liposomes. OMI is a linear unsaturated fatty acid with an amphiphilic structure consisting of a lipophilic octadecyl tail and a hydrophilic carboxyl head portion. These long unsaturated carboxylate complexes could increase the permeability on the cell membrane through the "perturbation" effect, resulting in much more intercellular uptake of active CDDP and OCP-L. In addition, endocytosis was one of the pathways to internalize OCP-L into A549/ CDDP cells. Therefore, OCP-L could cross the cellular membrane in an "invisible" form that prevents the CDDP from being recognized by efflux pumps. The results from both ICP-MS and high-content analysis demonstrated that CDDP carried by OCP-L could be efficiently delivered into A549/CDDP cells.

In addition to the effect of OMI on cellular uptake, the insertion of the cis-unsaturated fatty chain of OMI will also affect CDDP efflux due partly to their influence on the expression of ATP7B. Overexpression of ATP7B was one of the major mechanism by which cancer cells developed MDR against CDDP. Evidences have demonstrated that the reduction of ATP7B expressions in CDDP-resistant tumor cells can prevent CDDP from pumping out. ${ }^{20,21}$ Our data demonstrated a significantly reduced expression level of ATP7B after treatment with OP-L and OCP-L (Figure 3A, C). The main reason was that OMI-CDDP-N was synthesized through acid-labile monocarboxylato and an $\mathrm{O} \rightarrow \mathrm{Pt}$

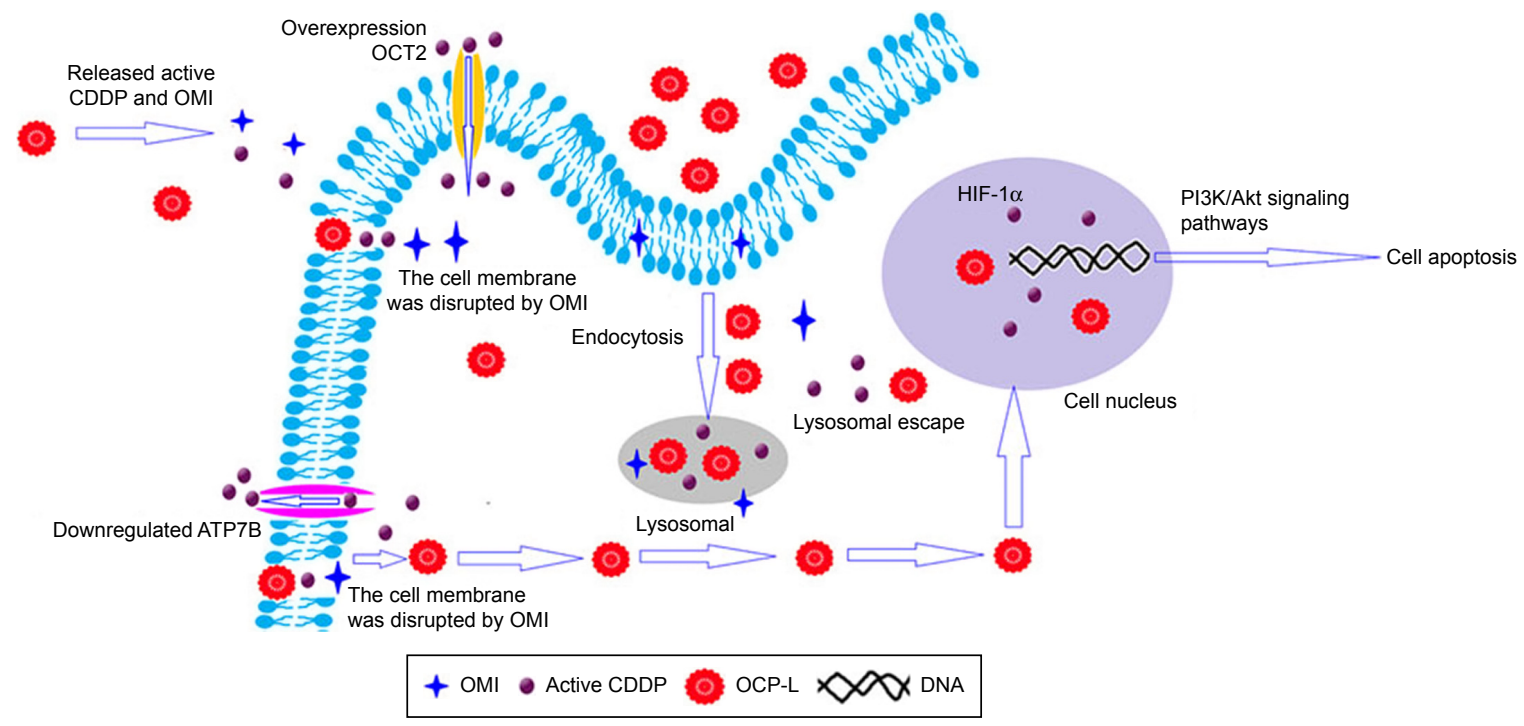

Figure 4 Possible mechanisms for overcoming resistance in A549/CDDP cells using OCP-L.

Abbreviations: A549/CDDP, CDDP-resistant human lung cancer cells; Akt, protein kinase B; ATP7B, ATPase copper-transporting beta polypeptide; CDDP, cisplatin; HIF-I $\alpha$, hypoxia-inducible factor I $\alpha$-subunit; OCP-L, 3-octadecylcarbamoylacrylic acid-CDDP nanocomplexes-based liposomes; OCT2, organic cation transporter 2; OMI, 3-octadecylcarbamoylacrylic acid. 
coordinate bond, which could efficiently release charged active platinum and OMI into cytoplasm. However, Andersson et al have stated that this charged active platinum could not increase the expression of efflux pump. ${ }^{22}$ Moreover, the lipophilic octadecyl tail of OMI had a notable inhibition effect on the expression of ATP7B.

The OCP-L was internalized in A549/CDDP cells through caveolin-mediated endocytosis pathway and macropinocytosis. Once uptaken into the acidic environment of the endosome, the OCP-L could release sufficient intracellular active platinum and OMI because of the acidlabile monocarboxylato and an $\mathrm{O} \rightarrow \mathrm{Pt}$ coordinate bond. The released OMI improved the endosomal membrane permeability by inducing transient pores on the endosomal membrane. After destabilization with the endosomal membrane, the OMI and active CDDP might be efficiently released into the cytoplasm.

HIF- $1 \alpha$ has been recently recognized as a novel mediator of MDR. ${ }^{23,24}$ Ye et al ${ }^{18}$ have reported that HIF-1 $\alpha$ was overexpressed in both messenger RNA (mRNA) and protein levels in the A549/CDDP cells. Compared to negative control, OP-L markedly inhibited the expression of HIF-1 $\alpha$ $(P<0.05)$, suggesting that OMI could depress the HIF-1 $\alpha$ function to some extent. Compared to OP-L, the expression of HIF-1 $\alpha$ increased after treatment with OCP-L, which indicated that the released active platinum had a positive effect on the expression of HIF-1 $\alpha$. In contrast, the cells treated with CDDP-S showed the highest level of HIF-1 $\alpha$, revealing that the mechanisms of MDR reversal in A549/CDDP cells by OCP-L could attribute to the inhibition of HIF-1 $\alpha$ expression. It is worthwhile to point out that OCP-L enhanced the cytotoxicity against A549/CDDP cells. This may be attributed to the following reasons: 1) the cellular uptake of OCP-L was efficient; 2) the $\mathrm{pH}-$ responsive release manner of OCP-L could maximally reduce CDDP degradation outside the cells; 3) OMI-CDDP-N complexes were synthesized through a monocarboxylato and $\mathrm{O} \rightarrow \mathrm{Pt}$ coordination, which was more efficient than CDDP because of the rapid hydrolysis; 4) the restoration effect on OCT2 expression and inhibition effect on ATP7B expression of OCP-L further increased intracellular active platinum concentration and enhanced cytotoxicity; and 5 ) the inhibition effect of OCP-L on HIF-1 $\alpha$ and p-Akt level could increase the apoptotic of A549/CDDP cells.

\section{Conclusion}

In summary, OMI-CDDP-N-loaded liposomes were manufactured to reverse the MDR of CDDP. OCP-L exhibited OMI-dependent intracellular uptake and sustained nuclear retention on A549/CDDP cells. The MDR of OCP-L was reversed successfully against different mechanisms, including the restoration of the function of OCT2 to increase the uptake of CDDP, inhibiting the function of ATP7B to reduce efflux of CDDP, and inhibiting the activity of HIF- $1 \alpha$ and p-Akt to recover the block apoptosis pathway. These results demonstrated that the OCP-L would be a promising drug delivery system for reversing the MDR of lung cancer.

\section{Acknowledgments}

This work was supported by the Project of Innovation Team of University from the Education Department of Liaoning, People's Republic of China (LT201023) and the Beijing Sinuoer Biotech Co., Ltd., People's Republic of China. Nanjing KeyGEN Biotech Co., Ltd. is thanked for helping with the supply of necessary equipment required to achieve cell experiments.

\section{Disclosure}

The authors report no conflicts of interest in this work.

\section{References}

1. Wu D, Li L, Yan W. Knockdown of TC-1 enhances radiosensitivity of non-small cell lung cancer via the Wnt/beta-catenin pathway. Open Biol. 2016;5(4):492-498.

2. Chen $\mathrm{W}$, Zheng R, Zeng H, Zhang S. Epidemiology of lung cancer in China. Thoracic Cancer. 2015;6(2):209-215.

3. Oberoi HS, Nukolova NV, Kabanov AV, Bronich TK. Nanocarriers for delivery of platinum anticancer drugs. Adv Drug Delivery Rev. 2013; 65(13):1667-1685.

4. Amable L. Cisplatin resistance and opportunities for precision medicine. Pharmacol Res. 2016;106:27-36.

5. Mamot C, Drummond DC, Hong K, Kirpotin DB, Park JW. Liposomebased approaches to overcome anticancer drug resistance. Drug Resist Updates. 2003;6(5):271-279.

6. Zeng F, Ju RJ, Li XT, Lu WL. Advances in investigations on the mechanism of cancer multidrug resistance and the liposomes-based treatment strategy. J Pharma Invest. 2014;44(7):493-504.

7. Krieger ML, Eckstein N, Schneider V, et al. Overcoming cisplatin resistance of ovarian cancer cells by targeted liposomes in vitro. Int $J$ Pharmaceut. 2010;389(1-2):10-17.

8. Casagrande N, De Paoli M, Celegato M, et al. Preclinical evaluation of a new liposomal formulation of cisplatin, lipoplatin, to treat cisplatinresistant cervical cancer. Gynecol Oncol. 2013;131(3):744-752.

9. Carvalho Júnior AD, Vieira FP, De Melo VJ, et al. Preparation and cytotoxicity of cisplatin-containing liposomes. Braz J Med Biol Res. 2007; 40(8):1149-1157.

10. Zamboni WC, Gervais AC, Egorin MJ, et al. Systemic and tumor disposition of platinum after administration of cisplatin or STEALTH liposomal-cisplatin formulations (SPI-077 and SPI-077 B103) in a preclinical tumor model of melanoma. Cancer Chemoth Pharm. 2004; 53(4):329-336

11. Kim ES, Lu C, Khuri FR, et al. A phase II study of STEALTH cisplatin (SPI-77) in patients with advanced non-small cell lung cancer. Lung Cancer. 2001;34(3):427-432.

12. Song J, Xu TT, Zhang Y, et al. 3-octadecylcarbamoylacrylic acid-cisplatin nanocomplexes for the development of novel liposome formulation. Drug Deliv. Epub 2016 Apr 19. 
13. Cia P, Liu XY, Han FS. Establishment of human lung adenocarcinoma cisplatin-resistant cell line A549/DDP and the mechanism of it's drug resistance. Chinese J Lung Cancer. 1995;1(14):169-170.

14. Chauhan SC, Vannatta K, Ebeling MC, et al. Expression and functions of transmembrane mucin MUC13 in ovarian cancer. Cancer Res. 2009; 69(3):765-774.

15. Kerr MC, Teasdale RD. Defining macropinocytosis. Traffic. 2009; 10(4): 364-371.

16. Salomon JJ, Gausterer JC, Yahara T, et al. Organic cation transporter function in different in vitro models of human lung epithelium. Eur $J$ Pharm Sci. 2015;80:82-88.

17. Gao G, Wang J, Shi S. Expression of copper-transporting P-type adenosine triphosphatase (ATP7B) correlates with cisplatin-resistance in human lung adenocarcinoma cell line A549. Chinese J Lung Cancer. 2009;12(4):350-353. Chinese.

18. Ye MX, Zhao YL, Li Y, et al. Curcumin reverses cis-platin resistance and promotes human lung adenocarcinoma A549/DDP cell apoptosis through HIF-1 $\alpha$ and caspase-3 mechanisms. Phytomedicine. 2012;19(8-9): $779-787$
19. Gao Y, Li W, Liu X, Gao F, Zhao X. Reversing effect and mechanism of soluble resistance-related calcium-binding protein on multidrug resistance in human lung cancer A549/DDP cells. Mol Med Rep. 2015;11(3): 2118-2124.

20. Sancho-Martinez SM, Prieto-Garcia L, Prieto M, López-Novoa JM, López-Hernández FJ. Subcellular targets of cisplatin cytotoxicity: an integrated view. Pharmacol Ther. 2012;136(1):35-55.

21. Zisowsky J, Koegel S, Leyers S, et al. Relevance of drug uptake and efflux for cisplatin sensitivity of tumor cells. Biochem Pharmacol. 2007;73(2):298-307.

22. Andersson A, Hedenmalm H, Elfsson B, Ehrsson H. Determination of the acid dissociation constant for $\mathrm{cis}$-diammineaquachloroplatinum(II) ion A hydrolysis product of cisplatin. J Pharm Sci. 1994;83(6):859-862.

23. Lin HH, Tsai CW, Chou FP, et al. Andrographolide down-regulates hypoxia-inducible factor-1alpha in human non-small cell lung cancer A549 cells. Toxicol Appl Phar. 2011;250(3):336-345.

24. Rohwer N, Cramer T. Hypoxia-mediated drug resistance: novel insights on the functional interaction of HIFs and cell death pathways. Drug Resist Updates. 2011;14(3):191-201.

\section{Publish your work in this journal}

Drug Design, Development and Therapy is an international, peerreviewed open-access journal that spans the spectrum of drug design and development through to clinical applications. Clinical outcomes, patient safety, and programs for the development and effective, safe, and sustained use of medicines are the features of the journal, which has also been accepted for indexing on PubMed Central. The manuscript management system is completely online and includes a very quick and fair peer-review system, which is all easy to use. Visit http://www.dovepress.com/testimonials.php to read real quotes from published authors.

Submit your manuscript here: http://www.dovepress.com/drug-design-development-and-therapy-journal 